\title{
openheart Tocilizumab increases citrullinated histone 3 in non-ST segment elevation myocardial infarction
}

\author{
Ragnhild Helseth (D) , ${ }^{1}$ Ola Kleveland, ${ }^{2}$ Thor Ueland, ${ }^{3,4,5}$ Rune Wiseth, ${ }^{2}$ \\ Jan Kristian Damas, ${ }^{6,7}$ Kaspar Broch, ${ }^{8}$ Annika Michelsen, ${ }^{3,4}$ Bjørn Bendz (D) , 8 \\ Lars Gullestad,,$^{3,5,8,9}$ Pål Aukrust, ${ }^{3,4,10,11}$ Ingebjørg Seljeflot ${ }^{1,3}$
}

To cite: Helseth R, Kleveland 0 , Ueland T, et al. Tocilizumab increases citrullinated histone 3 in non-ST segment elevation myocardial infarction. Open Heart 2021;8:e001492. doi:10.1136/ openhrt-2020-001492

Received 22 0ctober 2020 Revised 1 March 2021 Accepted 12 April 2021
Check for updates

(c) Author(s) (or their employer(s)) 2021. Re-use permitted under CC BY-NC. No commercial re-use. See rights and permissions. Published by BMJ.

For numbered affiliations see end of article.

Correspondence to Dr Ragnhild Helseth; ragnhild. helseth@gmail.com

\section{ABSTRACT}

Objective Beyond reducing inflammation and troponin $\mathrm{T}(\mathrm{TnT})$ release, the interleukin-6 receptor antagonist tocilizumab reduces neutrophil counts in patients with non-ST segment elevation myocardial infarction (NSTEMI). It is unclear if this is related to formation of neutrophil extracellular traps (NETs), carrying inflammatory and thrombotic properties.

Methods In a placebo-controlled trial, 117 patients with NSTEMI were randomised to a single dose of tocilizumab $(n=58)$ or placebo $(n=59)$ before coronary angiography. The NETs related markers double-stranded DNA (dsDNA), myloperoxidase-DNA (MPO-DNA) and citrullinated histone 3 (H3Cit) were measured at five consecutive time points during hospitalisation (days 1-3).

Results Our major findings were: (1) H3Cit levels were significantly higher in the tocilizumab compared with the placebo group at all time points (all $p<0.05$ ), and H3Cit area under the curve (AUC) was 2.3 fold higher in the tocilizumab compared with placebo group $(p<0.0001)$. (2) MP0-DNA and dsDNA did not differ between the groups. (3) In both treatment arms, dsDNA AUC was associated with TnT AUC. (4) Neutrophil count AUC correlated inversely to H3Cit AUC $(p=0.015)$ in the total population. Conclusions In patients with NSTEMI, treatment with tocilizumab is associated with increased circulating H3Cit levels, suggesting that tocilizumab enhances NETosis. Further studies should clarify whether NETosis is a relevant side effect of tocilizumab. Regardless of tocilizumab, dsDNA associated with TnT release, indicating a link between extracellular nuclear material and myocardial injury.

\section{INTRODUCTION}

The clinical benefit of inhibiting inflammatory mediators in coronary artery disease (CAD) has recently been demonstrated in clinical trials, ${ }^{2}$ and the inflammatory pathway from interleukin-1 (IL-1) to IL-6 and $\mathrm{C}$ reactive protein (CRP) has been identified as a central axis. ${ }^{3}$ In 2016 , we reported that a single dose of the IL-6 receptor (IL-6R) antagonist tocilizumab attenuated the inflammatory response, as assessed by CRP, in patients with non-ST segment elevation myocardial

\section{Key questions}

What is already known about this subject?

- Administration of the interleukin-6 receptor antagonist tocilizumab has recently been demonstrated to attenuate inflammation and troponin T release in patients with non-ST segment elevation myocardial infarction. In tocilizumab-treated patients, a dramatic reduction in circulating neutrophils has been observed. On neutrophil activation, neutrophil chromatin studded with neutrophil proteins can be related in to the extracellular space and form neutrophil extracellular traps (NETs). Any effect of interleukin-6 receptor antagonism on NETs formation is unclear.

What does this study add?

- Treatment with tocilizumab is associated with increased circulating markers of NETs, suggesting that interleukin-6 receptor antagonism might enhance NET formation.

How might this impact on clinical practice?

- Further studies should clarify if NETosis is a relevant side effect of tocilizumab treatment in patients with non-ST segment elevation myocardial infarction.

infarction (NSTEMI). Circulating troponin $\mathrm{T}$ (TnT) was reduced, particularly in patients who underwent percutaneous coronary intervention (PCI). ${ }^{4}$ In patients treated with tocilizumab, we observed a significant fall in circulating neutrophils. The implication of this finding was, however, not further investigated.

During activation, neutrophils are able to expel parts of their nuclear content decorated with a range of cytoplasmatic and granular proteins into the extracellular space to form spindle-like structures called neutrophil extracellular traps (NETs). ${ }^{5}$ During NETs formation, or NETosis, an important event preceding chromatin decondensation is citrullination of specific amino acids. The resultant citrullinated histones are regarded as relatively specific markers of NETs, as are 
complexes of double-stranded DNA (dsDNA) and the neutrophil granule protein myeloperoxidase (MPODNA complexes). Extracellular dsDNA has also widely been used as a marker of NETs, although its specificity is questionable in the context of non-neutrophil cell death. Whereas NETs seem to have beneficial antimicrobial properties, they have also been reported to be prothrombotic and cytotoxic. ${ }^{6}{ }^{7}$ A prothrombotic NETs phenotype has repeatedly been shown in coronary thrombi from patients with acute myocardial infarction $(\mathrm{MI})^{8-10}$ and is associated with myocardial injury as measured by troponin levels and MRI. ${ }^{11} 12$

As NETs have been suggested as stimulators of the IL-1/ IL-6 inflammatory axis, ${ }^{3}$ and potential also vice versa, we hypothesised that the anti-inflammatory and potentially cardioprotective effect of tocilizumab in patients with NSTEMI could be mediated through reduced NETosis. In this study, we aimed to explore whether treatment with tocilizumab affected circulating markers of NETs. We also assessed whether these markers were associated with myocardial injury as assessed by TnT.

\section{MATERIALS AND METHODS \\ Study design}

Details of the study design have been published previously. ${ }^{4}$ In brief, this randomised, double-blind, placebocontrolled trial was designed to evaluate the effect of a single dose of the anti-IL-6R antibody tocilizumab in NSTEMI. The trial was conducted at two high-volume PCI centres in Norway. Patients between 18 and 80 years with NSTEMI who were scheduled for coronary angiography were eligible for inclusion. Exclusion criteria included significant non-CAD cardiac disease, clinical instability, medications or diseases affecting inflammation, contraindications to tocilizumab, or any other condition that could interfere with protocol adherence. Patients were randomised in a 1:1 fashion to receive a single intravenous infusion of tocilizumab $280 \mathrm{mg}$ or placebo prior to coronary angiography.

\section{Patient and public involvement}

It was not appropriate or possible to involve patients or the public in the design, or conduct, or reporting, or dissemination plans of this study.

\section{Blood sampling protocol}

Blood samples were drawn at five time points during hospitalisation (day 1: baseline and evening, day 2: morning and evening, day 3: morning), and again after 3 months. Peripheral venous blood was drawn into endotoxinfree blood collection tubes with EDTA as anticoagulant (plasma) and with no additives (serum). The EDTA tubes were immediately placed on melting ice and centrifuged within $30 \mathrm{~min}$ at $>2500 \mathrm{~g}$ for $20 \mathrm{~min}$ to obtain platelet-poor plasma. Serum was centrifuged at $>2100 \mathrm{~g}$ for $15 \mathrm{~min}$ after full coagulation $(\sim 45 \mathrm{~min})$ at room temperature. Immediately following centrifugation, the plasma and serum were frozen and stored at $-80^{\circ} \mathrm{C}$ in multiple aliquots until analyses.

\begin{tabular}{|c|c|c|c|c|}
\hline & Total population $(n=117)$ & Placebo $(n=59)$ & Tocilizumab ( $n=58)$ & $P$ value \\
\hline Age, years, mean (SD) & $60(8.9)$ & $60.1(9.9)$ & $59.8(7.7)$ & 0.859 \\
\hline Female, $n(\%)$ & $14(12.0)$ & $5(8.5)$ & $9(15.5)$ & 0.241 \\
\hline Body mass index, $\mathrm{kg} / \mathrm{m}^{2}$, mean (SD) & $28.1(4.0)$ & $27.4(4.4)$ & $28.8(3.3)$ & 0.055 \\
\hline Hypertension, n (\%) & $43(36.8)$ & $17(28.8)$ & $26(44.8)$ & 0.072 \\
\hline Diabetes mellitus, n (\%) & $21(17.9)$ & $10(16.9)$ & $11(19)$ & 0.776 \\
\hline Current smoking, $\mathrm{n}(\%)$ & $32(27.6)$ & $17(28.8)$ & $15(26.3)$ & 0.763 \\
\hline Previous myocardial infarction, $\mathrm{n}(\%)$ & $16(13.7)$ & $7(11.9)$ & $9(15.5)$ & 0.565 \\
\hline $\begin{array}{l}\text { Symptom onset to inclusion, days, median }(25,75 \\
\text { percentiles) }\end{array}$ & $2(1,3)$ & $2(1,3)$ & $2(2,3.5)$ & 0.197 \\
\hline Maximum TnT, ng/L, median ( 25,75 percentiles) & $271(134,935)$ & $271(143,800)$ & $315(134,1064)$ & 0.867 \\
\hline PCl, n (\%) & $88(75.2)$ & $47(79.7)$ & $41(70.7)$ & 0.261 \\
\hline Aspirin, $n(\%)$ & $116(99.1)$ & $59(100)$ & $57(98.3)$ & 0.496 \\
\hline Clopidogrel, n (\%) & $64(54.7)$ & $32(54.2)$ & $32(55.2)$ & 0.919 \\
\hline Ticagrelor, $\mathrm{n}(\%)$ & $53(45.3)$ & $27(45.8)$ & $26(44.8)$ & 0.919 \\
\hline Low molecular weight heparin, n (\%) & $105(90.5)$ & $54(91.5)$ & $51(89.5)$ & 0.706 \\
\hline Statin, $n(\%)$ & $106(90.6)$ & $53(89.8)$ & $53(91.4)$ & 0.774 \\
\hline Betablocker, n (\%) & $90(76.9)$ & $45(76.3)$ & $45(77.6)$ & 0.866 \\
\hline Neutrophil count, $10^{9} / \mathrm{L}$, median $(25,75$ percentiles) & $4.8(3.4,6.1)$ & $4.5(3.4,6.0)$ & $5.1(3.4,6.4)$ & 0.471 \\
\hline
\end{tabular}

Numbers are given as mean (SD), median (25, 75 percentiles) or numbers (\%), as appropriate.

$P$ value refers to difference between the placebo and tocilizumab groups.

$\mathrm{PCl}$, percutaneous coronary intervention; $\mathrm{TnT}$, troponin $\mathrm{T}$. 


\section{Laboratory analyses}

Citrullinated histone 3 (H3Cit) was analysed by a commercial sandwich ELISA kit (Cayman Chemical, Ann Arbor, Michigan, USA) and performed according to the manufacturer. Serum in dilution 1:2 in ELISA buffer was used. Intra-assay and inter-assay coefficients of correlations $(\mathrm{CVs})$ were $6.2 \%$ and $8.3 \%$, respectively. MPO-DNA complexes were assessed in undiluted serum, using an ELISA technique previously described by Kessenbrock $e t$ $a l .{ }^{13}$ Briefly, the capture antibody anti-MPO (AbD Serotec, Hercules, California, USA) was coated to plates and incubated overnight at $4^{\circ} \mathrm{C}$. After blocking with bovine serum albumine, patient samples and a peroxidaselabelled anti-DNA antibody (Cell Death Detection kit, Roche Diagnostics GmbH, Mannheim, Germany) were added. Finally, a peroxidase substrate was added and absorbance measured, expressed as optical density (OD) units with an intra-assay and inter-assay CVs of 5.2\% and $11.8 \%$, respectively. Levels of dsDNA were measured in EDTA plasma in dilution 1:10 in TRIS/EDTA buffer by a fluorescent nucleic acid stain, Quant-iT PicoGreen (Invitrogen, Paisley, UK) using fluorometry (Fluoroskan Ascent, Thermo Fisher Scientific Oy, Vantaa, Finland). The intra-assay and inter-assay CVs were $2.4 \%$ and $6.0 \%$, respectively.

High-sensitivity CRP was analysed on a MODULAR platform (Roche Diagnostics, Basel, Switzerland), and high-sensitivity TnT was measured by an electrochemiluminescence immunoassay (ELICA; Elecsys 2010 analyzer, Roche Diagnostics). Leucocytes with subgroups were counted consecutively using routine methods at the hospitals' clinical biochemistry laboratories.

\section{Statistics}

Demographic data are given as mean (SD), median (25, 75 percentiles) or numbers (\%) as appropriate. As H3Cit, dsDNA and MPO-DNA all were unevenly distributed, non-parametric statistics were used in analyses concerning these markers. Group differences were analysed by the Mann-Whitney $U$ test, independent-samples t-test or $\chi^{2}$ test as appropriate, while within group changes in the NETs markers were analysed by the Wilcoxon's signed rank test. Correlation analyses were performed by Spearman's rho. The area under the curve (AUC) was calculated from the five measurements from baseline to day 3 for dsDNA, MPO-DNA, H3Cit, neutrophils and TnT. TnT was logarithmically transformed before calculation of the AUC for consistency with the publication of the trial's primary endpoint. ${ }^{4}$ The level of statistical significance was set to $<0.05$. All statistical analyses were performed in IBM SPSS, V.26.

\section{RESULTS}

\section{Baseline characteristics}

Baseline characteristics for the total study cohort are shown in table 1 . As outlined, no significant differences according to treatment allocation were observed.

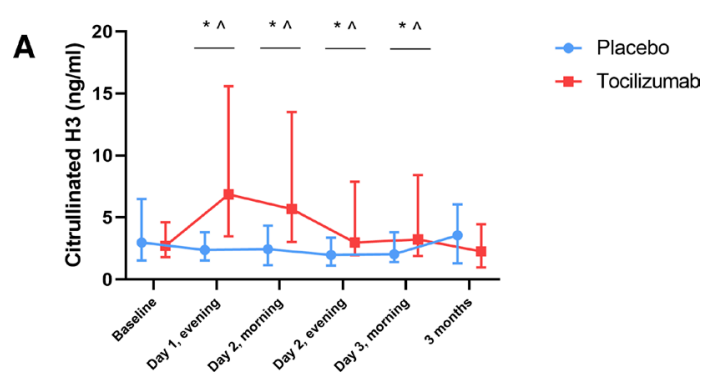

B
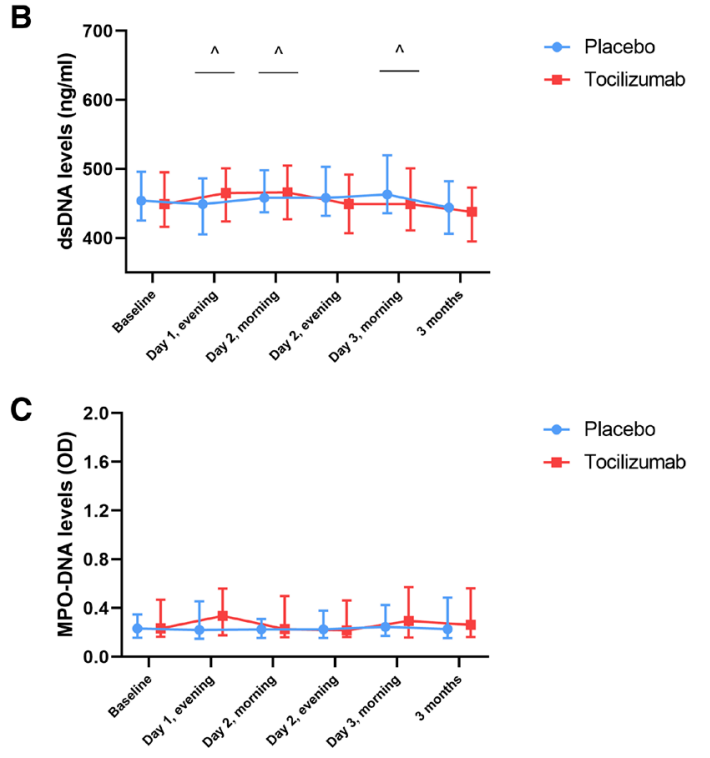

D

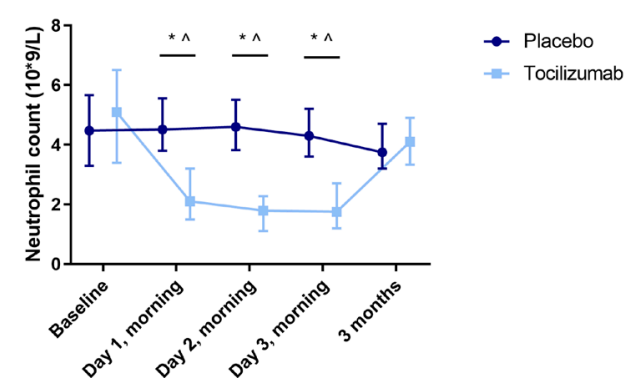

Figure 1 NETs levels and neutrophil count in according to treatment with tocilizumab or placebo during hospitalisation. The figure is made using GraphPad Prism 8. Statistical test: Mann-Whitney $U$ test. Values are given as medians (25, 75 percentiles). * Significant difference between groups at corresponding time points $(p<0.05)$. 'Significant difference in change from baseline $(p<0.05)$. dsDNA, double-stranded DNA; MPO-DNA, myloperoxidase-DNA; NETs, neutrophil extracellular traps.

No patients in either group underwent coronary artery bypass grafting within the three first days of hospital admission. In particular, neutrophil cell count did not differ between the groups (table 1).

\section{Markers of NETs across treatment arms}

Serum levels of H3Cit increased significantly more and were significantly higher in patients treated with tocilizumab than in patients treated with placebo during the first 3 days after randomisation (figure 1A). The AUC for H3Cit was 2.3 times higher in the tocilizumab group 
Table 2 NETs marker AUCs according to time of inclusion and revascularisation by $\mathrm{PCl}$ or not

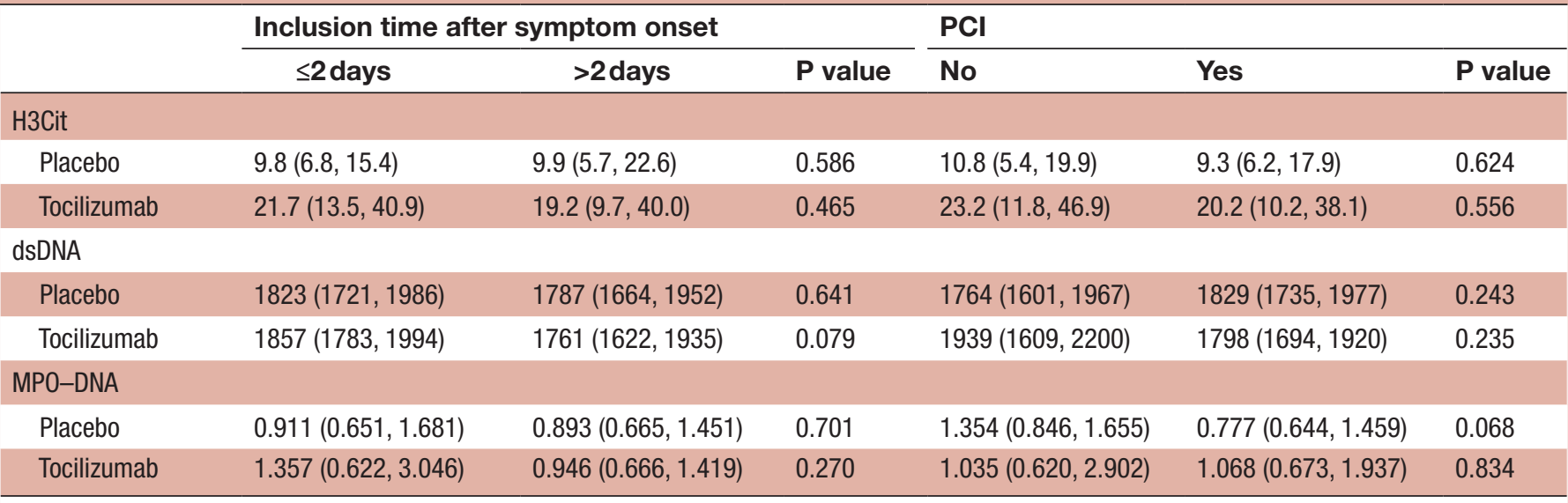

Numbers are given as medians (25, 75 percentiles).

AUC, area under the curve; dsDNA, double-stranded DNA; H3Cit, citrullinated histone 3; MPO-DNA, myeloperoxidase-DNA; NETs, neutrophil extracellular traps; $\mathrm{PCI}$, percutaneous coronary intervention.

compared with the placebo group (9.87 vs $23.15 \mathrm{ng}$ / $\mathrm{mL} \cdot$ days, $\mathrm{p}<0.0001)$. Plasma levels of dsDNA did not consistently differ between the treatment groups (figure 1B). Levels of MPO-DNA did not differ between the groups at any time (figure 1C). Moreover, the AUCs for dsDNA and MPO-DNA did not differ significantly between the treatment groups (tocilizumab vs placebo: $1817 \mathrm{vs} 1823 \mathrm{ng} / \mathrm{mL} \cdot$ days, $\mathrm{p}=0.926$ for dsDNA and 1.065 vs $0.893 \mathrm{OD}$-days, $\mathrm{p}=0.323$ for MPO-DNA). Results were unchanged when including the 3 months' values in the AUC calculations (data not shown).

The levels of the NETs markers had similar time profiles when patients were stratified by time from symptom onset to inclusion ( $\leq 2$ days vs $>2$ days), and by revascularisation by PCI $(n=88)$ or not. Neither inclusion time nor revascularisation by PCI affected the AUCs of the NETs markers (table 2).

\section{Markers of NETs and myocardial injury}

dsDNA AUC correlated significantly to peak TnT in the total population and in the placebo group, although not in the tocilizumab group (table 3). dsDNA AUC also correlated with TnT AUC in the total population and in both single groups (table 3). Neither H3Cit nor MPO-DNA AUCs correlated to peak TnT or TnT AUC (table 3).

\section{Markers of NETs and the neutrophil cell count}

The time profile of neutrophil counts is shown in figure 1D. As outlined in figure 1D, tocilizumab treatment

Table 3 Correlations between peak TnT, AUCs of TnT and neutrophil cell count, and NETs markers the first 3 days after randomisation

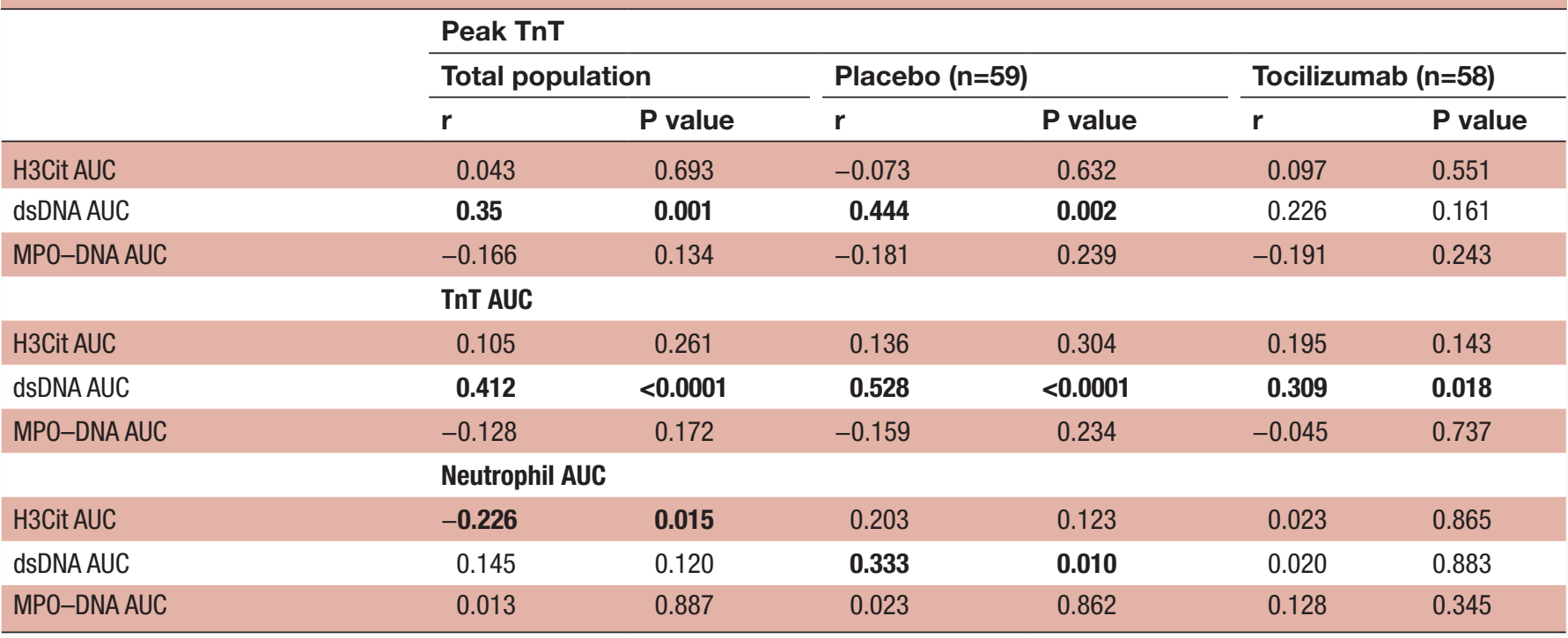

Significant values are marked in bold

AUC, area under the curve; dsDNA, double-stranded DNA; H3Cit, citrullinated histone 3; MPO-DNA, myeloperoxidase-DNA; NETs, neutrophil extracellular traps; TnT, troponin T. 
was associated with a steep fall in neutrophils. Neutrophil counts were significantly lower in the tocilizumab group at all measured times during hospital admission after randomisation ( $\mathrm{p}<0.0001$ for all). The AUC of H3Cit, but not those of dsDNA and MPO-DNA, correlated inversely to the AUC of the neutrophil cell count in the total population (table 3).

\section{DISCUSSION}

In this subanalysis of the randomised, placebo-controlled trial on patients with NSTEMI, treatment with the antiIL-6R antibody tocilizumab was associated with an increase in serum levels of H3Cit, suggesting tocilizumab to enhance NETosis. This process did not affect circulating levels of TnT. However, levels of dsDNA were significantly associated with TnT release, regardless of tocilizumab treatment, suggesting a possible link between extracellular nuclear material and myocardial injury that is not attenuated by tocilizumab.

Opposed to our initial hypothesis, patients with NSTEMI who were allocated to a single dose of tocilizumab had increased levels of H3Cit, indicating that tocilizumab enhanced NETosis. A transient tocilizumabinduced neutropaenia has previously been reported, where possible mechanisms have been suggested to include enhanced margination of circulating neutrophils and impaired myelopoiesis. ${ }^{14}$ Apoptosis as the cause of neutropaenia and high H3Cit levels in this cohort could be relevant. Arguments against this include that H3Cit is regarded specific to NETosis, that circulating levels of H3Cit do not seem to parallel circulating markers of apoptosis and necrosis, ${ }^{1516}$ and, indeed, previous studies have suggested that administration of tocilizumab does not seem to affect neutrophil apoptosis. ${ }^{14}$ A theoretic explanation for the findings could be that tocilizumab stimulates neutrophil adhesion to the endothelial wall, resulting in neutrophil/endothelial activation, triggering NETosis. Nonetheless, our present data do not imply that the beneficial effect of tocilizumab on TnT release in NSTEMI patients involves impaired NETosis. In fact, the increase in H3Cit in the tocilizumab group should be further investigated as a potential harmful effect of this medication.

Whereas the NETs marker levels were not associated with TnT in the tocilizumab group, dsDNA levels in the total cohort correlated with peak TnT and TnT AUC. These observations are well in line with several previous reports where circulating NETs markers, in particular dsDNA, associate with MI size. ${ }^{11} 1217$ The underlying pathophysiology for this association is unclear. Previous reports indicate NETs participation in ischaemia reperfusion injury and direct cytotoxic effects of extracellular histones as possible pathways. ${ }^{18-21}$ Whether dsDNA is a potent NETs surrogate marker and not merely a marker of cardiomyocyte cell death is still unanswered, thus these correlations should be interpreted with some caution. First, there was no overall difference in dsDNA between the two treatment groups. Moreover, the association between peak TnT and dsDNA was only seen in the placebo group and was not related to PCI. Nonetheless, these data also underscore that the effect of tocilizumab and its clinical consequences should be further investigated.

Our study has several limitations. First, the number of patients and the infarct sizes were modest. Second, the time from symptom onset to the administration of the study drug varied. Third, the inconsistent observations for the three investigated markers of NETs underscore the ongoing discussion and strive to map specific circulating surrogate markers of NETs. Currently, there is no consensus on this matter. Finally, we have reported on associations, not causal relationships. Whether neutrophils exposed to tocilizumab induce NETs in the setting of NSTEMI was not investigated in the current study, but will be an important follow-up of our findings.

\section{CONCLUSION}

Administration of a single dose of the IL-6R antagonist tocilizumab in this cohort of NSTEMI patients associated with increased circulating levels of the NETs marker H3Cit, suggesting that tocilizumab might enhance NETosis. Although this observation was not associated with myocardial injury as measured by TnT, further studies should clarify if NETosis is a side effect of tocilizumab that has clinical relevance in patients with MI.

\section{Author affiliations}

${ }^{1}$ Center for Clinical Heart Research, Department of Cardiology, Oslo University Hospital Ullevål, Oslo, Norway

${ }^{2}$ Clinic of Cardiology, St. Olavs Hospital, Trondheim, Norway

${ }^{3}$ Institute of Clinical Medicine, University of Oslo, Oslo, Norway

${ }^{4}$ Research Institute of Internal Medicine, Oslo University Hospital Rikshospitalet, Oslo, Norway

${ }^{5}$ K.G. Jebsen Center for Cardiac Research, University of 0slo, 0slo, Norway ${ }^{6}$ Department of Infectious Diseases, St Olavs Hospital, Trondheim, Trondheim, Norway

${ }^{7}$ Centre of Molecular Inflammation Research, Department of Clinical and Molecular Medicine NTNU, Trondheim, Norway

${ }^{8}$ Department of Cardiology, Oslo University Hospital Rikshospitalet, Oslo, Norway

${ }^{9}$ Center for Heart Failure Research, Oslo University Hospital, Oslo, Norway

${ }^{10}$ Section of Clinical Immunology and Infectious Disease, Oslo University Hospital Rikshospitalet, 0slo, Norway

${ }^{11} \mathrm{~K} . \mathrm{G}$. Jebsen Thrombosis Research and Expertise Center, University of Troms $\emptyset$, Tromsø, Norway

Acknowledgements We thank the laboratory staff, especially Jeanette K.Steen at Center for Clinical Heart Research (CCHR), Oslo University Hospital Ullevål for excellent laboratory assistance.

Contributors RH, PA and IS have planned the work described in this article. The NETs analyses have been performed by the laboratory staff at CCHR, Department of Cardiology, Oslo University Hospital Ullevål. RH have written drafts of the manuscript. OK, TU, RW, JKD, KB, AM, BB, LG, PA and IS have all contributed to the interpretation of the results and finalisation of the manuscript. $\mathrm{RH}$ is guarantor of the overall manuscript content.

Funding The work has been funded by unrestricted grants from Stein Erik Hagen's Foundation for Clinical Heart Research, 0slo, Norway.

Competing interests None declared.

Patient consent for publication Not required. 
Ethics approval The study was approved by the Regional Committee for Medical and Health Research Ethics of South-Eastern Norway, the Norwegian Medicines Agency, the Data Protection Representative at the two participating centerscentres, and is registered at ClinicalTrials.gov, NCT 01491074. The study was conducted according to the Helsinki Declaration. All participants gave informed, written consent for participation.

Provenance and peer review Not commissioned; externally peer reviewed.

Data availability statement All data are in a repository at Department of Cardiology, Oslo University Hospital and are available upon reasonable request.

Open access This is an open access article distributed in accordance with the Creative Commons Attribution Non Commercial (CC BY-NC 4.0) license, which permits others to distribute, remix, adapt, build upon this work non-commercially, and license their derivative works on different terms, provided the original work is properly cited, appropriate credit is given, any changes made indicated, and the use is non-commercial. See: http://creativecommons.org/licenses/by-nc/4.0/.

ORCID iDs

Ragnhild Helseth http://orcid.org/0000-0001-5012-9214

Bjørn Bendz http://orcid.org/0000-0002-1392-6481

\section{REFERENCES}

1 Ridker PM, Everett BM, Thuren T, et al. Antiinflammatory therapy with canakinumab for atherosclerotic disease. N Engl J Med 2017;377:1119-31.

2 Tardif J-C, Kouz S, Waters DD, et al. Efficacy and safety of low-dose colchicine after myocardial infarction. N Engl J Med 2019;381:2497-505.

3 Ridker PM. From C-reactive protein to interleukin-6 to interleukin-1: moving upstream to identify novel targets for Atheroprotection. Circ Res 2016;118:145-56.

4 Kleveland O, Kunszt G, Bratlie M, et al. Effect of a single dose of the interleukin- 6 receptor antagonist tocilizumab on inflammation and troponin T release in patients with non-ST-elevation myocardial infarction: a double-blind, randomized, placebo-controlled phase 2 trial. Eur Heart J 2016;37:2406-13.

5 Brinkmann V, Reichard U, Goosmann C, et al. Neutrophil extracellular traps kill bacteria. Science 2004;303:1532-5.

6 Fuchs TA, Brill A, Duerschmied D, et al. Extracellular DNA traps promote thrombosis. Proc Natl Acad Sci U S A 2010;107:15880-5.

7 Engelmann B, Massberg S. Thrombosis as an intravascular effector of innate immunity. Nat Rev Immunol 2013;13:34-45.

8 Mangold A, Alias S, Scherz T, et al. Coronary neutrophil extracellular trap burden and deoxyribonuclease activity in ST-elevation acute coronary syndrome are predictors of ST-segment resolution and infarct size. Circ Res 2015;116:1182-92.

9 Riegger J, Byrne RA, Joner M, et al. Histopathological evaluation of thrombus in patients presenting with stent thrombosis. A multicenter European study: a report of the prevention of late stent thrombosis by an interdisciplinary global European effort Consortium. Eur Heart J 2016;37:1538.1-49.

10 Novotny J, Chandraratne S, Weinberger T, et al. Histological comparison of arterial thrombi in mice and men and the influence of $\mathrm{Cl}$-amidine on thrombus formation. PLoS One 2018;13:e0190728.

11 Helseth $\mathrm{R}$, Solheim S, Arnesen $\mathrm{H}$, et al. The time course of markers of neutrophil extracellular traps in patients undergoing revascularisation for acute myocardial infarction or stable angina pectoris. Mediators Inflamm 2016;2016:1-8.

12 Helseth R, Shetelig C, Andersen Geir Øystein, et al. Neutrophil extracellular trap components associate with infarct size, ventricular function, and clinical outcome in STEMI. Mediators Inflamm 2019;2019:1-10.

13 Kessenbrock K, Krumbholz M, Schönermarck U, et al. Netting neutrophils in autoimmune small-vessel vasculitis. Nat Med 2009;15:623-5.

14 Wright HL, Cross AL, Edwards SW, et al. Effects of IL-6 and IL-6 blockade on neutrophil function in vitro and in vivo. Rheumatology 2014;53:1321-31.

15 Leshner M, Wang S, Lewis C, et al. PAD4 mediated histone hypercitrullination induces heterochromatin decondensation and chromatin unfolding to form neutrophil extracellular trap-like structures. Front Immunol 2012;3:307.

16 Jackson Chornenki NL, Coke R, Kwong AC, et al. Comparison of the source and prognostic utility of cfDNA in trauma and sepsis. Intensive Care Med Exp 2019;7:29.

17 Hofbauer TM, Mangold A, Scherz T, et al. Neutrophil extracellular traps and fibrocytes in ST-segment elevation myocardial infarction. Basic Res Cardiol 2019;114:33.

18 Ge L, Zhou X, Ji W-J, et al. Neutrophil extracellular traps in ischemiareperfusion injury-induced myocardial no-reflow: therapeutic potential of DNase-based reperfusion strategy. Am J Physiol Heart Circ Physiol 2015;308:H500-9.

19 Savchenko AS, Borissoff JI, Martinod K, et al. VWF-mediated leukocyte recruitment with chromatin decondensation by PAD4 increases myocardial ischemia/reperfusion injury in mice. Blood 2014;123:141-8.

20 Saffarzadeh M, Juenemann C, Queisser MA, et al. Neutrophil extracellular traps directly induce epithelial and endothelial cell death: a predominant role of histones. PLoS One 2012;7:e32366.

$21 \mathrm{Xu}$ J, Zhang X, Pelayo R, et al. Extracellular histones are major mediators of death in sepsis. Nat Med 2009;15:1318-21. 